Résumés des conférences et travaux

\title{
Nécrologies
}

\section{Louis Bazin (1920-2011)}

\section{Nicolas Vatin}

\section{(2) OpenEdition \\ Journals}

Édition électronique

URL : https://journals.openedition.org/ashp/2106

DOI : 10.4000/ashp.2106

ISSN : 1969-6310

Éditeur

Publications de l'École Pratique des Hautes Études

\section{Édition imprimée}

Date de publication : 1 septembre 2018

Pagination : XVII-XIX

ISSN : 0766-0677

\section{Référence électronique}

Nicolas Vatin, «Louis Bazin (1920-2011)", Annuaire de l'École pratique des hautes études (EPHE), Section des sciences historiques et philologiques [En ligne], 149 | 2018, mis en ligne le 06 juillet 2018, consulté le 03 août 2021. URL : http://journals.openedition.org/ashp/2106 ; DOI : https://doi.org/10.4000/ashp. 2106 


\section{LOUIS BAZIN \\ (1920-2011)}

N.B. : hommage prononcé en assemblée de section mais encore inédit.

Louis Bazin nous a quittés le 20 mars 2011.

Né à Caen le 29 décembre 1920, il était resté très attaché à ses origines normandes, dont témoignait son sens de l'humour. Brillant élève, il fut reçu en 1939 à l'École normale supérieure. L'agrégation de grammaire obtenue, il se spécialisa, en linguiste, dans l'étude du turc, sous la direction du grand Jean Deny auquel il vouait une profonde admiration. En 1943, il fut recruté comme attaché de recherche au CNRS. Cette époque de formation, vécue dans Paris occupé, n'était pas gaie. Mais Louis Bazin était jeune, et jeune marié : il n'en conserva pas que de mauvais souvenirs. Soucieux toujours, d'ailleurs, de ne pas assombrir les réunions amicales qu'il animait volontiers de sa joie de vivre, il préférait rappeler qu'il avait pu, dans ces temps involontairement écologiques, pêcher des écrevisses sous un pont de la Seine.

La guerre n'était pas achevée qu'il était envoyé en Turquie par le gouvernement français, en avril 1945. Installé à Ankara, où il enseignait le français à la faculté des sciences politiques, il y approfondit sa connaissance du turc et des Turcs.

À son retour en 1949, à moins de trente ans, il succéda à Jean Deny comme professeur délégué, puis titulaire (en 1957) à l'École des langues orientales. Sa thèse d'État soutenue en 1972, il devint en 1978 maître de conférences, puis en 1980 professeur à l'université Paris-III, à laquelle avaient été rattachées les Langues' O. C'est ainsi qu'au long de plus de trois décennies, il inculqua le turc à de nombreuses générations de turcologues. Tous ses anciens élèves, moi le premier, conservent un souvenir très fort de son enseignement qui, en trois ans, et avec l'aide bien entendu de ses collaborateurs, nous donnait sans douleur, dans le même temps, des bases grammaticales solides et les moyens de lire et de communiquer. Depuis 1968, les étudiants disposent de son Introduction à l'étude pratique de la langue turque qui, sous le couvert d'un titre modeste, est un modèle de grammaire pratique, faisant appel à l'intelligence de façon à la fois limpide, complète et concise. Le cours enrichissait ces connaissances de base, sans lourdeur, avec un emploi réfléchi de l'humour et des anecdotes qui ancrait à jamais les phénomènes importants dans les esprits.

Parallèlement à cet enseignement d'initiation, Louis Bazin assuma, dès 1950, une charge de directeur d'études dans notre IV e section de l'École pratique des hautes études, en remplacement de Jean Sauvaget. La lecture des rapports qu'il ne manqua jamais de faire parvenir fidèlement permet de suivre l'évolution de sa recherche et de sa réflexion, qu'on retrouve bien entendu dans sa bibliographie. On est d'abord frappé par l'étendue de ses curiosités et de ses compétences turcologiques, qui le faisaient aller de la langue des inscriptions « runiformes » du VIII ${ }^{\mathrm{e}}$ siècle aux parlers contemporains, du Haut Ienisseï ou du monde kirghize à l'Anatolie. Encore prenait-il en compte la nécessité de sortir du seul domaine turcologique, fût-ce à l'aide d'amis spécialistes, pour profiter de l'apport des sources chinoises ou sogdiennes. 
James Hamilton, dans la préface du recueil d'articles de Louis Bazin paru en 1994 sous le titre Les Turcs. Des mots, des hommes, distinguait trois axes majeurs dans son œuvre. Le premier, consistant dans l'étude des inscriptions et autres documents des Turcs anciens, est fondamental. Louis Bazin consacra son premier séminaire aux Hautes Études à l'épigraphie turque ancienne ( $\mathrm{VIII}^{\mathrm{e}}-\mathrm{X}^{\mathrm{e}}$ siècles) et y revint maintes fois par la suite, au fur et à mesure des nouvelles découvertes et publications, revenant sur les lectures de ses prédécesseurs ou les siennes propres et reconsidérant leurs interprétations. Le second axe est celui des systèmes chronologiques des Turcs anciens et médiévaux, sujet traité également à plusieurs reprises dans son séminaire et qui fut celui de sa thèse d'État, livre majeur sur les systèmes de calendriers des peuples turcophones de la Chine aux Balkans sur une durée de huit siècles, fondé sur une vaste documentation éparse qu'il lui avait fallu reconstituer et sur des calculs astronomiques et astrologiques. Soutenue en 1972, la thèse remaniée parut en 1991 sous le titre Les systèmes chronologiques dans le monde turc ancien. Le troisième axe est la question fondamentale des rapports linguistiques du turc et du mongol, sur lequel les résumés du séminaire de Louis Bazin, mais aussi la postface qu'il rédigea pour son recueil d'articles, montrent son souci de ne jamais rien tenir pour acquis et sa recherche perpétuelle de pistes neuves, qui l'amenèrent, avec l'aide d'Altan Gokalp, à recourir à l'informatique.

En définissant ces trois axes, James Hamilton soulignait à juste titre combien Louis Bazin était, profondément et avant tout sans doute, un linguiste. Ses travaux attestent du reste son intérêt pour la phonologie, la syntaxe ou les questions lexicales. Néanmoins, la langue n'était pour lui qu'un élément parmi d'autres de l'aventure humaine des peuples türks. Louis Bazin aimait les mots et c'est pourquoi, à l'évidence, il était un amateur éclairé de contrepets. Mais derrière les mots il aimait retrouver les hommes, leurs titres et leur organisation sociale, leurs croyances, leurs rapports avec la nature et le monde animal. C'est aussi comme documents historiques fournissant une information unique en son genre que les inscriptions anciennes l'intéressaient. Il prêtait une grande attention aux questions ethnologiques et folkloriques, mais également littéraires, le tout étant indissolublement lié. Aussi consacra-t-il nombre d'heures de son séminaire à étudier des textes littéraires de tous ordres, de toutes langues turkes, écrits ou oraux. Je citerai l'épopée kirghize de Manas, ou la geste de Dede Korkut, texte d'Anatolie orientale de l'époque des Moutons Blancs étudié dans son intégralité à deux reprises et dont il a publié, avec Altan Gokalp, l'unique traduction française.

Faute de la compétence requise, je préfère laisser à d'autres le soin d'analyser savamment l'œuvre de Louis Bazin pour revenir d'un mot sur sa pratique. S'il était un pédagogue, il avait aussi une grande force d'entraînement. Les travaux que j'ai évoqués, et dont j'ai pieusement recherché la trace dans l'Annuaire de notre section, portent tous la marque de la personnalité de Louis Bazin, mais aussi celle de son ouverture aux intérêts, questions et compétences de ses amis et collègues. J'ai souvent cité James Hamilton, Américain fixé à Paris, dont la collaboration de sinisant, notamment, fut précieuse. Sa mort brutale dans la cour du Collège de France fut pour Louis Bazin un coup terrible. Il faut aussi citer Pertev Nailî Boratav, grand spécialiste turc du folklore, dont la participation fut non moins essentielle, Altan Gokalp 
l'ethnologue, passionné comme lui par les astres et les monstres, Rémy Dor l'ethnolinguiste, qui partagea avec les auditeurs du séminaire le résultat de ses recherches sur les Kirghizes du Haut Pamir afghan...

Au-delà de ce cercle étroit de complices, le séminaire au cours du temps attira un nombre croissant de personnes intéressées par les mondes turks. Louis Bazin, qui avait relancé l'Institut d'études turques, avait fondé en 1967 une équipe de recherches associée au CNRS intitulée « Études turques » dont les membres, pour beaucoup ses anciens élèves, trouvaient profit à assister aux séminaires du lundi matin, qui devint bientôt le lieu de réunion hebdomadaire de la turcologie parisienne. Au sein de la section 44 du CNRS (vouée aux langues et civilisations orientales), Louis Bazin jouait en outre un rôle de premier plan dans l'organisation de la recherche. Cette carrière fut couronnée par son élection à l'Académie des inscriptions et belles-lettres en 1993.

Les dernières années furent assombries par la mort de sa femme Micheline et par la maladie qui le contraignit peu à peu à s'éloigner du milieu académique où il avait tenu une si grande place. Ceux qui furent ses élèves n'oublient pas cependant le grand savant qu'il fut, et plus encore l'homme toujours courtois et tolérant, disponible, discrètement attentif, sachant quand il le fallait apporter à ceux qui lui paraissaient le mériter le soutien de son influence.

Nicolas VATIN 